\title{
Cavity Ring-Down with OTDR for remote sensing
}

\author{
D. J. Passos, S. O. Silva, M. B. Marques, O. Frazão \\ INESC Porto, Rua do Campo Alegre 687, 4169-007 Porto, Portugal \\ Faculdade de Ciências da Universidade do Porto, Rua do Campo Alegre 687, Porto, Portugal
}

\begin{abstract}
This work presents a demonstration of the potential of fiber cavity ring-down for remote sensing, by using an OTDR to send impulses down $\sim 20 \mathrm{~km}$ of optical fiber at the end of which the fiber ring cavity was placed. The OTDR showed almost no losses in the fiber, so other ring-down cavities could be spliced along the same fiber. To study the sensitivity of the cavity ring an intensity sensor based on a taper was placed in the ring and glued to a translation stage. A displacement of the stage imposes a curvature on the taper and an associated loss. The configuration had a sensitivity of $(11.8 \pm 0.5) \mu \mathrm{s} / \mathrm{mm}$.
\end{abstract}

\section{INTRODUCTION}

The cavity ring down spectroscopy (CRDS) is a widely used technology for chemical and molecular analysis in real time [1]. The evolution of this technique led to the development of diverse configurations based on the same underlying principle, i.e., a resonant optical cavity with high reflectivity mirrors.

The recent development of a fiber optic based CRD, which uses a fiber loop as resonant cavity, has been gaining popularity in the community, for presenting a potential alternative to the usual CRD [2]. In this manner, the conceptual study of the CRD technique became a focus of attention in the area of optical spectroscopy, for the last decade. However, only recently, in 2004, has this technique been applied to the measurement of physical parameters, such as: deformation [3], [4], pressure [5], and temperature [6]. Still, biochemical sensing has been the main topic of this area of research. Several schemes based in the CRD technique in optical fiber have been implemented, such as: for gas detection [7], liquid phase [8], unicellular organisms [9], detection of xylene and cyclohexane [10] and, recently, the measurement of rhodamine $6 \mathrm{G}$ in aqueous solution [11].

The CRD technique has brought the development of refractive index sensors with some proposed configurations in the last five years [12]. In 2008, Ni et al. [13] used a long period grating as a resonant cavity in the fiber loop and studied the ring down decay time as a function of the changes in environment refractive index. Zhou et al. presented a tilted fiber Bragg grating as a sensing element, obtaining a maximum sensitivity of $154 \mu \mathrm{s} / \mathrm{RIU}$ and a resolution of $6.5 \times 10^{-6}$ RIU. The same research group fabricated a microchannel $(4.5 \mu \mathrm{m}$ in diameter) in the fiber loop, by means of a femtosecond laser, for detection of gels with different refractive indices [15]. This configuration revealed sensitivity superior to 300 $\mu \mathrm{s} / \mathrm{RIU}$ in the 1.37-1.448 and a resolution of $1.4 \times 10^{-4} \mathrm{RIU}$. Recently, Wong et al. [16] integrated in the resonant cavity a Mach-Zehnder interferometer based on photonic crystal fibers for detection of liquid with different refractive indices. In this case the maximum resolution was $7.8 \times 10^{-5}$ RIU.

This kind of new systems shows the possibility of obtaining high sensitivities and resolution in gas detection, providing the means for the development of a new generation of optical fiber sensors with strong applications on biochemical sensing and sensors in biological systems.

This work presents the potential of fiber CRD for remote sensing by using an Optical Time Domain Reflectometer (OTDR). To introduce the losses inside of the cavity a fiber taper is used as a displacement sensor. 


\section{EXPERIMENTAL RESULTS}

The experimental configuration of the CRD with an OTDR is presented in Figure 1. A commercial OTDR was used to send $1 \mu$ s impulses down an optical fiber (SMF 28) which, after an extension of around $20 \mathrm{~km}$, couples to a fiber loop through a (99:1) directional coupler. The $1 \mathrm{~km}$ fiber loop forms the ring cavity where the pulse will propagate, with a second (99:1) coupler and a photodetector used for measuring the decay of the pulse. Finally, the measured signal was observed in a commercial oscilloscope.

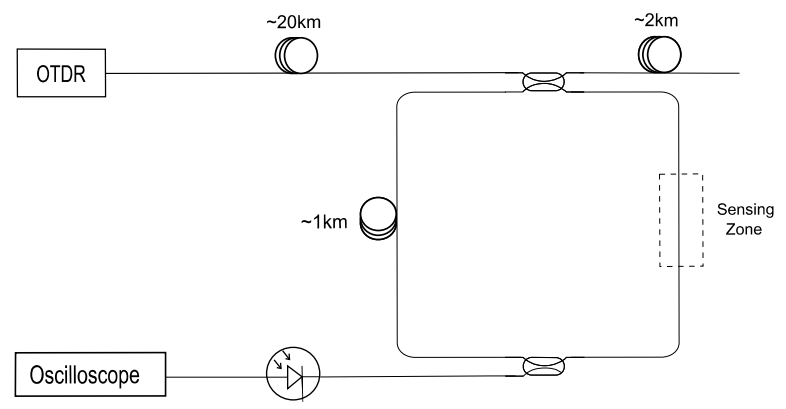

Figure 1: The cavity ring-down setup. The signal will be introduced by the OTDR and the monitoring will be done with a photodetector combined with an oscilloscope.

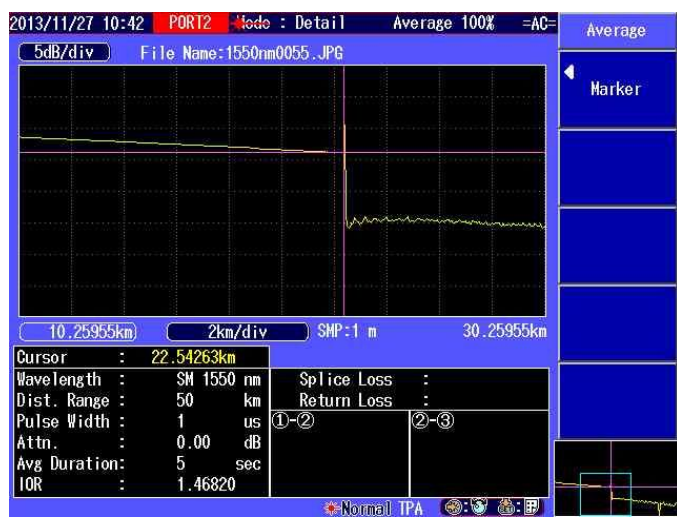

Figure 2: OTDR display while operating.

The OTDR graph (Figure 2) shows no measurable losses at the insertion point, presenting the possibility for additional CRD elements to be used along the same fiber. These results are expected due to the coupling ratio of 99:1. The OTDR parameters used in this experiment are: $0 \mathrm{~dB}$ attenuation, average duration of $5 \mathrm{sec}$ and a pulse width of $1 \mu \mathrm{s}$.

The measured signal at the oscilloscope is presented in Figures 3. The output intensity of the pulse was determined from a simple exponential function with the following equation: $I(t)=I_{0} \exp (-t / \tau)$, where $I_{0}$ is the initial intensity of the input pulse, $\tau$ is the decay time (ring-down time), which is the corresponding time when $I(t)$ decreases to $1 / e$ times of $I_{0}$. An exponential fit was done to obtain a ring-down time of $32 \mu \mathrm{s}$. This decay time changed appreciably upon introduced losses, for example, on bending/displacement of the fiber. 

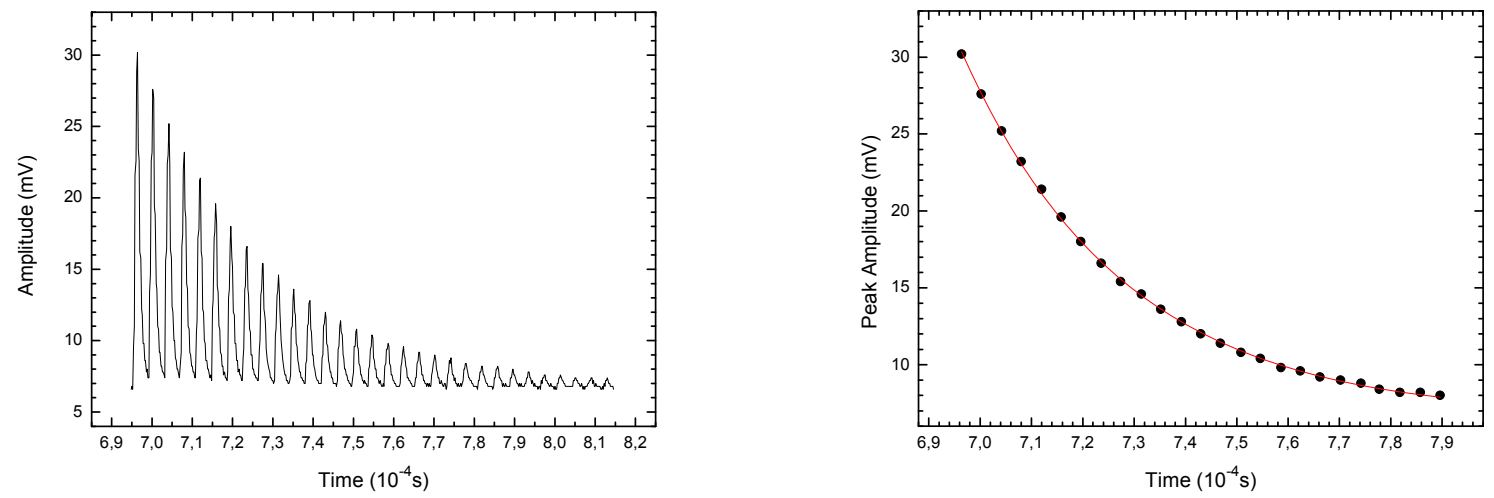

Figures 3: a) The decay of the pulse in the cavity, due to insertion losses in. Averages were taken to produce a clearer curve; b) An exponential fit of the peak amplitudes in the previous graph, from which the decay time is calculated.

To explore the sensitivity of the CRD setup, an intensity sensor was introduced in the ring cavity. The sensor was a 50 $\mu \mathrm{m}$ diameter taper, placed on a translation stage. The displacement of the fixed fiber end forces the taper to bend, inducing measurable losses. The previous analysis was reproduced for several displacement values. The different associated losses led to different decay times (Fig. 4a), with a linear dependence (Fig. 4b). The linear fit gave a sensitivity of $(11.8 \pm 0.5) \mu \mathrm{s} / \mathrm{mm}$.
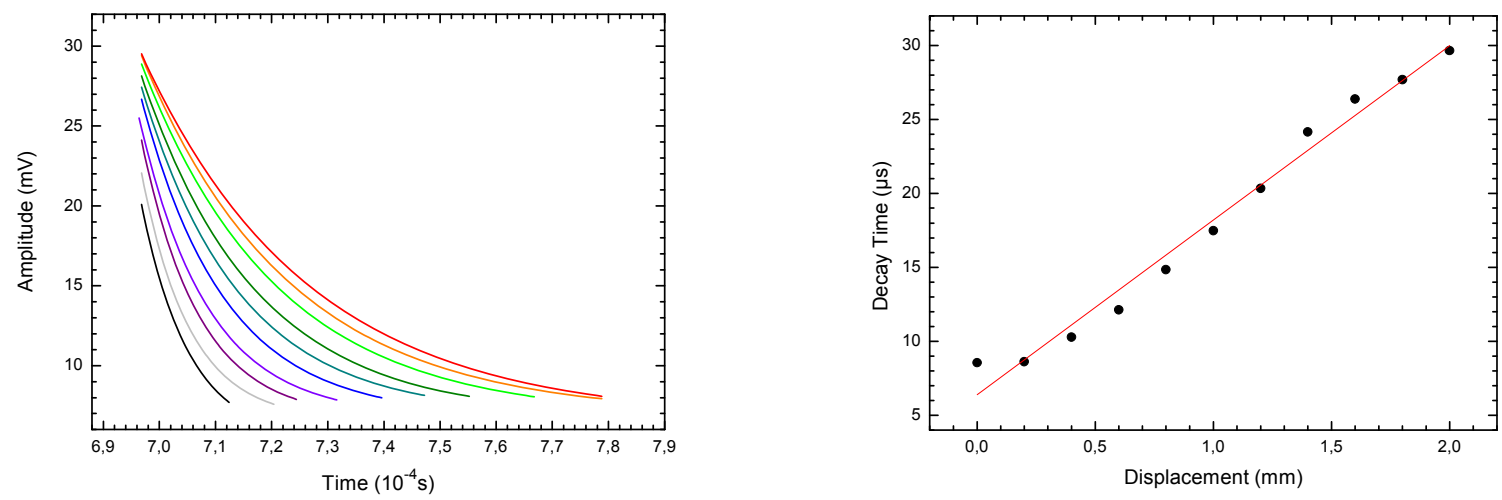

Figures 4: a) The exponential fitting curves of the decaying pulse for different introduced losses (through the bending of the taper); b) Decay times taken from previous exponential fits. A greater displacement here corresponds to an extended taper (state of lower loss)

\section{CONCLUSION}

It has been shown that an OTDR can be used to provide optical pulses to a fiber along which multiple fiber ring cavities could potentially be placed. The setup was demonstrated by the placement of a taper on a cavity and displacing the fixed end of the taper, forcing it to bend, and introducing losses that converted into a change in the decay time. This arrangement showed a sensitivity of $11.8 \pm 0.5 \mu \mathrm{s} / \mathrm{mm}$. The cavity ring-down technique is seen to hold some potential for remote sensing, through its implementation on optical fibers. 


\section{ACKNOWLEDGMENT}

Project NORTE-07-0124-FEDER-000058 is financed by the North Portugal Regional Operational Programme (ON.2 O Novo Norte), under the National Strategic Reference Framework (NSRF), through the European Regional Development Fund (ERDF), and by national funds, through the Portuguese funding agency, Fundação para a Ciência e a Tecnologia (FCT).

\section{REFERENCES}

[1] G. Berden, R. Peeters, and G. Meijer, "Cavity ring-down spectroscopy: Experimental schemes and applications," Int. Rev. Phys. Chem., vol. 19, pp. 565-607, 2000.

[2] G. Stewart, K. Atherton, and B. Culshaw, "Cavity-enhanced spectroscopy in fiber cavities," Opt. Lett., vol. 29, no. 5, pp. 442-444, 2004.

[3] W. P. Tarsa D. M. Brzozowski, P. Rabinowitz, and K. K. Lehmann, "Cavity ringdown strain gauge," Opt. Lett., vol. 29, no. 12, pp. 1339-1341, 2004.

[4] N. Ni, C. C. Chan, X. Y. Dong, J. Sun, and P. Shum, "Cavity ring-down long period fiber grating strain sensor," Meas. Sci. Technol., Special Issue, vol. 18, pp. 3135-3138, 2007.

[5] C. Wang and S. T. Scherrer, "Fiber ringdown pressure sensors," Opt. Lett., vol. 29, no. 4, pp. 352-354, 2004.

[6] C. Wang, "Fiber ringdown temperature sensor," Opt. Eng. Lett., vol. 44, no. 3, 030503, 2005.

[7] G. Stewart, K. Atherton, H. Yu, and B. Culshaw, "An investigation of an optical fibre amplifier loop for introcavity and ring-down cavity loss measurements," Meas. Sci. Technol., vol. 12, pp. 843-849, 2001.

[8] R. S. Brown, I. Kozin, Z. Tong, R. D. Oleschuk, and H.-P. Loock, "Fiber-loop ring-down spectroscopy," J. Chem. Phys., vol. 117, pp. 10444-10447, 2002.

[9] P. B. Tarsa, A. D. Wist, P. Rabinowitz, and K. K. Lehmanna, "Singlecell detection by cavity ring-down spectroscopy," Appl. Phys. Lett., vol. 85, no. 19, pp. 4523-4525, 2004.

[10] J. A. Barnes, M. Dreher, K. Plett, R. Brown, C. M. Crudden, H.-P. Loock, "Chemical sensor based on a long period fibre grating modified by a functionalized polymethylsiloxane coating," Analyst, vol. 133, pp. 1541$1549,2008$.

[11] C. M. Rushworth, D. James, J. W. L. Lee, and C. Vallance, "Top Notch Design for Fiber-Loop Cavity RingDown Spectroscopy,” Anal. Chem., vol. 83, no. 22, pp. 8492-8500, 2011.

[12] H. Waechter, J. Litman, A. H. Cheung, J. A. Barnes, and H.-P. Loock, "Chemical Sensing Using Fiber Cavity Ring-Down Spectroscopy,” Sensors, vol. 10, pp. 1716-1742, 2010.

[13] N. Ni, C. Chan, L. Xia, and P. Shum, "Fiber cavity ring-down refractive index sensor," IEEE Photon. Technol. Lett., vol. 20, no. 16, pp. 1351-1353, 2008.

[14] K. M. Zhou, D: Webb, M. Farries, N. Hayes, L. Zhang, and I. Bennion, "Biochemical sensor based on a novel all-fibre cavity ring down spectroscopy technique incorporating a tilted fibre Bragg grating," Opt. Lasers Eng., vol. 47, pp. 1023-1027, 2009.

[15] K. M. Zhou, D. J. Webb, C. B. Mou, M. Farries, N. Hayes, and I. Bennion, "Optical fiber cavity ring down measurement of refractive index with a microchannel drilled by femtosecond laser," IEEE Photonics Technol. Lett., vol. 21, no. 22, pp. 1653-1655, 2009.

[16] W. C. Wong, W. Zhou, C. C. Chan, X. Dong, and K. C. Leong, "Cavity ring-down refractive index sensor using photonic crystal fiber interferometer", Sens. Actuat. B, vol. 161, pp. 108-113, 2012. 